\title{
Necessity of Institutional Transitional Care Strategies
}

\author{
Mrs. Suchana Roy Bhowmik ${ }^{1}$, Dr.Alaka Deshpande ${ }^{2}$, Dr.Tapati Bhattacharjee ${ }^{3}$, \\ Dr.Prabha Dasila ${ }^{4}$ \\ ${ }^{1}$ Ph.D.Scholar, MGM Institute of Health Sciences, Navi Mumbai,India. \\ ${ }^{2}$ Honorary Professor of Medicine, Grant Medical College, Mumbai,India. \\ ${ }^{3}$ Professor, Bharti Vidya Peeth College of Nursing, Pune,India \\ ${ }^{4}$ Professor and Director, MGM Institute's University Department of Nursing, Navi Mumbai,India.
}

\begin{abstract}
:
Aim of the study is to assess the existing facility for the transitional care and necessity of formulation of institutional transitional care strategies.

Background: In a hospital set up addressing the needs of the ICU patients during transition and developing strategies for transitional care and implementing the same by nurses who play a crucial role. Care is planned based on individual patient needs. It is also recommended that the patients are adequately prepared for the change to facilitate the transitional care. Knowledge about transitional needs, empowerment and patienteducation seems to be important issues for facilitating transitions among ICU nurses.

Design: A descriptive study design was applied.

Methods: Non probability purposive sampling technique was used. A total of 50 nurses who are working in the ICUs participated in the study. A structured Questionnaire to assess the need of transitional care strategies in ICU is used for data collection.

Result: The finding significantly shows that among the study participants $96 \%$ and in the nursing population more than $85 \%$ felt that there is necessity of formulation of institutional transitional care strategies.

Conclusions: The finding illustrated that there is necessity of formulation of institutional transitional care strategies. This study emphasises that one of the most critical success factors in transitional care delivery is the role of nurse. If standardized transitional care strategies are available in the health care set up then sustainability of a positive transitional care outcome can be expected.

Relevance to clinical practice: Transitional care needs assessment in ICU by nurses underpins clinical practice. Preparing ICU nurses with this knowledge help them to applying this into practice.
\end{abstract}

\section{Introduction}

It has been observed that patients are admitted to the Intensive Care Unit (ICU) for a variety of reasons. Admission to an ICU is often a brief but extremely grim hospital experience to critically ill patients. Critically ill patients require close observation, continuous monitoring and prompt attention by critical care team which comprises of specially trained physicians, nurses and other professionals. Patients receive many types of treatment and various specialized and skilled nursing care in the ICU. Stay in the ICU may have both short- and long-term effects on overall recovery of patient. Once a person is able to breath unaided or needs minimal specialized care, he/she may be transferred from the ICU to a ward or high dependency unit. ${ }^{1-3}$ Transfer of patients from ICU to the other units is an everyday procedure which may be due to patient's recovery or the demand for ICU beds. ${ }^{2}$

The patient in the ICU set up is closely monitored and their every need attended by the health care workers. As the patient's condition remain fluctuating, it is difficult to say when he will be transferred to general wards. The patient in wards get general care but not the specialized one which he received in the ICU. The nurse and patient ratio in developing country is very unsatisfactory and demand for skilled nurses being enormous. The patient is admitted in ICU for serious ailments and he has undergone a closed monitoring. A severe psychological trauma occurs when he is transferred. He feels as if he is suddenly thrown into a common pool for which he is not prepared psychologically. Therefore transitional care is considered as part of quality care but due to various reasons it is not followed in our health care set up like overload of patients, inadequate nurses, lack of communication, lack of training of health care workers and lack of role of administration in the health care set up to implement transitional care strategies.

\section{Background}

Transition is a process in which something undergoes a change and passes from one state, stage, form, or activity to another. Critically ill patients in the intensive care unit (ICU) often experience multiple transitions, as they move from ICU to intermediate care unit, then to general ward. Each transition represents unique 
challenges for patient, their family members, and the healthcare professionals involved in the patients' care. ${ }^{4}$ Many studies supported that unplanned discharge affects physical and psychological parameters of patient. Compromised transitional care may result in complications, including adverse events, readmission and increased rates of mortality. ${ }^{5}$

ICU transitional care is the care provided before, during, and after the transfer of an ICU patient to another care unit. It should ensure minimal disruption and optimal continuity of care for the patient. This care may be provided by ICU nurses, acute care nurses, physicians, and other healthcare professionals. The main concern is to achieve a coordinated, strengthening, patient-centered standard of care to facilitate transition between different level of care. ${ }^{3}$

But it has been found that there is a gap within the health care set up, which is due to differences in the different level of care. These gaps are not always apparent, nor are appropriate bridging reactions clear. ${ }^{6}$ According to Stephanie C. and Fiona C., the discharge process for patients within the ICU setting is complicated and largely underappreciated. There are fundamental, misunderstood differences in prioritization and care of patients between the ward and ICU. ${ }^{7}$ Although critical care nurses are aware that, they are unable to deliver transitional care due to workload issues, unplanned discharges and inadequate communication. By understanding this challenge and to fill this gap. ICUs needs to adopt organized discharge plan strategies to improve patient care, improved interdepartmental communications which can be facilitated by organizational, individual and team work. ${ }^{8}$

Knowledge among nurses about transitional needs, empowerment and patient-education seems to be an important issue for facilitating transitions. ${ }^{9}$ Awareness and knowledge of patient's psychosocial needs are ignored and not identified by nurses for which many researches suggest that a comprehensive assessment tool is required. Chaboyer W, Kendall E, Kendall M, Foster M suggested in their study that there is a need for ICU nurses, ward nurses and affiliated healthcare professionals to provide an emotional support to patients throughout ICU transfer. It is understood that transitional care will prepare the patient and their families for smooth recovery from illness. Once they are adequately prepared, they will accept the change. ${ }^{10}$

So, to provide high quality care it is important to promote for more effective transition care including improvements in communication, adequate education, close follow up etc. There is clear evidence and wide agreement among healthcare providers/stakeholders that a standardized and policy-driven protocol was important to an effective discharge planning. In this regard researcher suggested that there should be strategies to provide this support must be developed, implemented and evaluated.

Aim of the study is to assess the existing facility for the transitional care and necessity of formulation of institutional transitional care strategies.

\section{Material and methods}

Total of 50 nurses working in the ICUs of a teaching hospital participated in the study. A descriptive study design was carried out and samples were selected with non probability purposive sampling technique. A 30 items structured Questionnaire was used to collect the data. The tool was developed by extensive review of literature and seeking opinion from experts. Reliability of the tool was calculated by using crohns's back i.e.914.

Ethical consideration: The project has been approved by the ethics committee of the institution. Informed consent was obtained from the participants before initiating the study.

\section{Results}

Table 1. Knowledge of participants about effects of transition on patients

\begin{tabular}{|l|l|l|}
\hline Category & Score Key & Frequency in \% \\
\hline High level of knowledge & $18-24$ & 88 \\
\hline Medium level of knowledge & $13-18$ & 12 \\
\hline No knowledge & $8-13$ & 0 \\
\hline Total & & $\mathbf{1 0 0}$ \\
\hline
\end{tabular}
transition .

Above table depicts that $88 \%$ study participants having high level of knowledge about effect of

Using this data and applying proportion test it is observed that more than $75 \%$ of the nursing population having high level of knowledge about effects of transition.

Table 2. Knowledge of participants about transitional care strategies

\begin{tabular}{|l|l|l|}
\hline Category & Score Key & Frequency in \% \\
\hline High level of knowledge & $21-27$ & 84 \\
\hline Medium level of knowledge & $15-21$ & 16 \\
\hline No knowledge & $9-15$ & 0 \\
\hline Total & & $\mathbf{1 0 0}$ \\
\hline
\end{tabular}



strategies.

Above table depicts that $84 \%$ study participants having high level of knowledge about transitional care

Using this data and applying proportion test it is observed that more than $70 \%$ of the nursing population having high level of knowledge about transitional care strategies.

Table 3. Agreement about importance of role of nurse in transitional care

$\mathbf{N}=\mathbf{5 0}$

\begin{tabular}{|l|l|l|}
\hline Category & Score key & Frequency in \% \\
\hline Strongly Agree & $\mathbf{1 7 - 2 1}$ & 74 \\
\hline Agree & $\mathbf{1 2}-\mathbf{1 7}$ & 26 \\
\hline Disagree & $\mathbf{7 - 1 2}$ & 0 \\
\hline Total & & $\mathbf{1 0 0}$ \\
\hline
\end{tabular}

Above table depicts that $74 \%$ study participants strongly agreeing that there is important role of nurse in transitional care.

Using this data and applying proportion test it is observed that more than $60 \%$ of the nursing population strongly agreeing that there is important role of nurse in transitional care.

Table 4. Agreement about implementation of transitional care strategies among the participants

\begin{tabular}{|l|l|l|}
\hline Category & Score key & Frequency in \% \\
\hline Strongly Agree & $14-18$ & 76 \\
\hline Agree & $10-14$ & 24 \\
\hline Disagree & $6-10$ & 0 \\
\hline Total & & $\mathbf{1 0 0}$ \\
\hline
\end{tabular}

$\mathbf{N}=\mathbf{5 0}$

Above table depicts that $76 \%$ study participants strongly agree that there should be implementation of transitional care strategies by the nurses in the institution.

Using this data and applying proportion test it is observed that more than $60 \%$ of the nursing population strongly agree there should be implementation of transitional care strategies by the nurses.

Table 5 : Necessity of formulation of institutional transitional care strategies $\quad \mathbf{N}=\mathbf{5 0}$

\begin{tabular}{|l|l|l|}
\hline Category & Score Key & Frequency in \% \\
\hline Less need & $4-7$ & 0 \\
\hline Moderate need & $7-10$ & 4 \\
\hline High need & $10-12$ & 96 \\
\hline Total & & $\mathbf{1 0 0}$ \\
\hline
\end{tabular}

Above table depicts that $96 \%$ study participants felt that there is necessity of formulation of institutional transitional care strategies.

Using this data and applying proportion test it is observed that more than $85 \%$ of the nursing population felt that there is necessity of formulation of institutional transitional care strategies.

Observation: Observations from study are as follows which after proportion test reveal the findings in general nursing population.

- Among the study participants $88 \%$ and in the nursing population more than $75 \%$ are having high level of knowledge about effect of transition on critically ill patients.

- Among the study participants $84 \%$ and in the nursing population more than $70 \%$ are having high level of knowledge about transitional care strategies.

- Among the study participants $74 \%$ and in the nursing population more than $60 \%$ are strongly agreeing that there is important role of nurse in transitional care.

- Among the study participants $76 \%$ and in the nursing population more than $60 \%$ are strongly agreeing that there should be implementation of transitional care strategies by the nurses in the institution.

- Among the study participants $96 \%$ and in the nursing population more than $85 \%$ felt that there is necessity of formulation of institutional transitional care strategies.

\section{Conclusion}

The findings of this study provide a new insight into the necessity of formulation of institutional transitional care strategies. This study emphasises that one of the most critical success factors in transitional care delivery is the role of the nurse. A good body of evidence shows that Advanced Practice Nurses involved in such programs and if there are standardization transitional care strategies facility then that will help in sustainability of patient care, by reducing re-hospitalizations, decrease costs, increase quality of life, and reduce 
mortality. ${ }^{12}$ Nurses' understanding of critically ill patients' transition may significantly impact the patients' care in the Intensive Care Unit. Thus, it emphasizes the need for formulation of transitional care strategies in the institution. But the crucial role played by the nursing staff in transitional care need to be updated by continued training and education.

Relevance to clinical practice: Transitional care needs assessment in ICU by nurses underpins clinical practice. Preparing and training ICU nurses with this knowledge help them to practice transitional care strategies which ensures quality care to the patient. Emphasizing implementation of transitional care strategies in the institution by all the health care workers will facilitate better quality care to the patients..

\section{References}

[1]. CPMC hand outs, Sutter health CPMC we plus you, retrieved from http://www.cpmc.org/learning/documents/icu-ws.html

[2]. Ramona O. Hopkins. Managing Post-Intensive Care Syndrome in the ICU: Long-Term Quality of Life in ICU Survivors. society of crotical care medicine:2013 - 4 August, retrieved from http://www.sccm.org/Communications/CriticalConnections/Archives/Pages/Strategies-Ensure-Quality-Life-Survivors.asp

[3]. Wendy Chaboyer, Heather James, and Melissa Kendall. Transitional Care After the Intensive Care Unit, Current Trends and Future Directions Crit Care Nurse June 2005. vol. 25 no. 3 16-28

[4]. N Ramakrishnan. Cost of intensive care in India: review article. Year : 2008 | Volume : 12 | Issue : 2 | Page : 55-61

[5]. Kate Field, Suman Prinjha, Kathy Rowan .One patient amongst many': a qualitative analysis of intensive care unit patients' experiences of transferring to the general ward. Critical Care 2008, 12:R21 doi:10.1186/cc6795

[6]. Richard C., Marta R., and David W. Gaps in the continuity of care and progress on patient safety, BMJ.2000;320:791

[7]. Stephanie C., and Fiona C,. Discharge practices for the intensive care patient: A qualitative exploration in the general ward setting. Intensive and Critical Care Nursing, Volume 30, Issue 5, October 2014, Pages 292-300.

[8]. Frances Lin, Wendy Chaboyer, Marianne Wallis, A literature review of organisational, individual and teamwork factors contributing to the ICU discharge process. Australian Critical Care, Volume 22, Issue 1, February 2009, Pages 29-43

[9]. Marie Häggström, Kenneth Asplund, and Lisbeth Kristiansen.Struggle with a gap between intensive care units and general wards, Int J Qual Stud Health Well-being. 2009; 4: 181-192. Published online Sep 1, 2009. http://www.ncbi.nlm.nih.gov

[10]. Chaboyer W, Kendall E, Kendall M, Foster M. Transfer out of intensive care: a qualitative exploration of patient and family perceptions. Aust Crit Care. 2005 Nov;18(4):138-41, 143-5.

[11]. Mary Naylor, and Stacen A. Keating, Transitional Care: Moving patients from one care setting to another. Am J Nurs. 2008 Sep; 108(9 Suppl): 58-63.

[12]. Bottoms, Holly, "Advanced Practice Nurses in Transitional Care: A Source to the Solution" (2014). Graduate Research Projects. Paper 1.http://knowledge.e.southern.edu/gradnursing/1 